\title{
Ten Possible Experiments on Communication and Deception*
}

\author{
Joel Sobel \\ University of California, San Diego
}

July 8, 2012

\begin{abstract}
I describe ten situations in which experimental data may provide useful guidance to the study of cheap-talk games. Journal of Economic Literature Classification Numbers: C92, C83; Keywords: communication, cheap talk, experiments, deception.
\end{abstract}

*Prepared for the Conference on "Deception, Incentives and Behavior" organized by Uri Gneezy and held at UCSD in April 2012. I am grateful to NSF for financial support and Andreas Blume, Vincent Crawford, David Cooper, Rachel Croson, Rick Harbaugh, and Bettina Rockenbach for comments and references. 


\section{Introduction}

This paper describes ten problems in which experimental data might help direct or constrain theories of communication.

Three themes that arise in the examples are the importance of a message space that has meaning exogenous to the game, the implications of heterogenous attitudes towards honesty, and the likelihood of non-trivial communication in which the Sender prefers the payoffs in the babbling equilibrium to those in a communicative equilibrium.

Throughout the paper, the underlying game involves one or more informed Senders who learn the state of nature, send a message or messages to one or more uninformed Receivers. The Receivers take an action that is payoff relevant to all players. Talk is cheap in the sense that the message does not enter directly into payoffs. Some games have additional choices for the players and some are sensitive to the possibility that preferences may depend on behavioral factors.

\section{Conflict of Interest over Equilibria}

Many papers (perhaps starting with Cooper, DeJong, Forsythe, and Ross (1992)) demonstrate that communication can improve coordination. Cai and Wang (2006)'s results suggest that theory predicts less communication than one observes in laboratory settings. Experimental studies of games in which the Receiver and Sender have different preferences over equilibria provide an opportunity to test the generality of these findings. In this section I describe an environment in which the Sender may play a babbling strategy even when revealing equilibria exist. Section 2 describes a different kind of example in which the babbling outcome may be robust.

Consider the situation described by the payoff table below. In the figures, there is a Row for each type of the Sender and a Column for each action of the Receiver. In each cell the first number is the Sender's payoff and the second number is the Receiver's payoff.

\begin{tabular}{|c||c|c|c|}
\hline & Home & Italian & French \\
\hline \hline French & 10,0 & 5,5 & $0,-10$ \\
\hline Italian & 10,0 & $0,-10$ & 5,5 \\
\hline
\end{tabular}

Here is a story. The Sender and Receiver are a couple. The Receiver despises a co-worker of the Sender (the Enemy). The Enemy is going out to a restaurant in the evening. The Sender knows the location (it is either a French restaurant or an Italian restaurant). The Receiver believes that the Enemy is equally likely to go to either. The couple can do one of three things for dinner: they can eat at home, go to the Italian restaurant, or go to the French restaurant. The Receiver would like to go out to dinner, provided that the Sender's enemy is not there, but the Receiver would rather stay home than face a .5 probability of meeting the Enemy. The Sender prefers to stay home, but prefers to go to the restaurant without the Enemy.

This game has a babbling equilibrium outcome in which the Sender sends an uninformative message and the Receiver chooses to stay home. It also has a fully revealing 
equilibrium in which the Receiver infers the Enemy's location and goes out to other restaurant. Plainly, the Sender prefers the babbling equilibrium and the Receiver prefers the informative equilibrium.

Is there a systematic tendency to play one equilibrium over another? As long as messages are available, the informative equilibrium exists. On the other hand, one might expect that the Receiver will not update the prior if the Sender is silent. An experimental design might vary whether the Sender can remain silent.

\section{Categorical Communication}

Increasing the complexity of the state space increases opportunities for effective communication. Experiments might clarify conditions under which players take advantage of the opportunity to communicate.

Imagine a cheap-talk game in which the only equilibrium outcome is babbling. For example, consider

\begin{tabular}{|c||c|c|}
\hline & Bad & Good \\
\hline \hline Low & 4,4 & $8, a$ \\
\hline High & 5,0 & 10,4 \\
\hline
\end{tabular}

where $a$ is a parameter. In this game the Sender knows the ability of a worker. Ex ante, the Receiver believes that the worker is equally likely to be high or low ability. The Receiver must assign the worker to a job. The Receiver wants to place a low-ability worker in a bad job and a high-ability worker is a good job. The Sender prefer the worker to be in a good job (regardless of the true quality of the worker). The only equilibrium of this game involves babbling. Receiver's optimal action is to always assign the worker to a bad job if $a<0$ and to a good job if $a>0$.

Now imagine that the Sender has information about two workers, whose abilities are independently and identically distributed. Chakraborty and Harbaugh (2007) provide the theory of categorical cheap talk that applies to this class of games. They identify conditions under which there exist informative equilibria in which the Sender identifies the better worker. ${ }^{1}$ In the example, suppose that the Sender can make statements of the form "worker $i$ is better than worker $j$." Provided that $a \in[-8 / 3,8 / 3]$ there is an equilibrium in which the Sender makes these statements honestly (identifying the better worker when exactly one is good and randomizing over the messages otherwise) and the Receiver responds by assigning the worker the Sender favors to the good job and the other worker to the bad job. ${ }^{2}$ There also exists a babbling equilibrium (whether the Receiver assigns both workers to the good job or to the bad job depends on whether $a$ is positive).

In the example, there is only a babbling equilibrium when the Sender reports on only one worker, but there may be an informative equilibrium (in addition to the babbling

\footnotetext{
${ }^{1}$ Chung and Harbaugh (2012) provide experimental evidence for categorical cheap talk.

${ }^{2}$ When $a>8 / 3$ the Receiver will assign both workers to the good job and when $a<-8 / 3$, the Receiver will assign both workers to the bad job.
} 
equilibrium) when the Sender reports on two workers. When $a \in(0,8 / 3)$, the Sender prefers the babbling equilibrium, while the Receiver prefers the informative equilibrium for all $a$. One would like to know whether players take advantage of the opportunity to make categorical statements and to what extent this depends on whether the players have the same preferences over equilibrium payoffs.

\section{Factors that Lead to Over-communication}

Experimental evidence suggests that agents are more honest and more credulous than equilibrium theory suggests. The mechanism underlying this phenomenon is uncertain.

There are two theories that may organize the findings. The first theory assumes that preferences depend on messages, leading to what typically is interpreted as costly lying. Charness and Dufwenberg (2006), Gibson, Tanner, and Wagner (forthcoming), Gneezy (2005), and Hurkens and Kartik (2009) provide evidence that agents are averse to lying. Charness and Dufwenberg (2006) hypothesize that guilt and shame make deception less likely. They provide a systematic way to test the importance of these effects.

The lying cost theories require a model that goes beyond the standard cheap-talk model in which messages are abstract quantities that have no meaning outside the model. Without the extension, there is no way to define lying. Understanding the lying cost theories requires identifying the how results are sensitive to exogenous labeling of messages.

All cheap-talk games have a babbling equilibrium. Therefore when a game has an informative equilibrium outcome, it will have multiple, qualitatively different equilibrium outcomes. Standard refinement arguments do not systematically reject the noninformative babbling equilibrium. In the standard one-dimensional cheap talk game studied by Crawford and Sobel (1982), there are now several different arguments (Chen (2006), Chen, Kartik, and Sobel (2008), Crawford and Sobel (1982), Gordon (2011), Kartik (2009), and Kartik and Sobel (2012)) that rule out the babbling outcome and make the equilibrium in which the Receiver takes the most actions the prediction with the strongest theoretical support. Kartik and Sobel's selection argument relies on agents sharing a common conception of the message space (in the sense that it is common knowledge that Senders associate higher messages to highest states and Receivers take higher actions in response to higher messages). Hence the argument suggests that communication will be more effective if Sender's and Receiver's share a common conception of the message space. One way to investigate this hypothesis is to compare behavior in games in which messages are abstract symbols that have no systematic connection to the action space or space of types to situations in which the type, message, and action spaces all share a common ordering.

Blume, DeJong, Kim, and Sprinkle (1998 and 2001) conduct experiments that compare games in which messages are abstract symbols (with no natural ordering) to more games in which messages have a natural ordering. They provide evidence that subjects can communicate effectively without a common language, but that the rate of convergence is more rapid when messages have natural meanings. These studies look at simple games. Games in which the Sender and Receiver have different preferences over equilibria provide another setting to test the hypothesis that providing subjects with a language 
that has a commonly understood exogenous meaning always enhances communication. For example, in the restaurant example of Section 1, will providing the Sender with the option to do nothing (that is, creating a message that conforms to the notion of "silence") reduce communication? In the case of categorical communication, would one find more separation in an environment where messages were labeled "the first worker is better" and "the second worker is better" than in one in which there was, in addition, a third message "both workers are good?"

Other authors organize observations about behavior in communication games by assuming that agents differ in their strategic sophistication. Cai and Wang (2006) and Wang, Spezio, and Camerer (2010) trace excessive honesty and credulity to level- $k$ thinking. Heterogeneity in the population plays a major role in both the level- $k$ story and in the lying cost models. In the case of level- $k$ theories, it is heterogeneity in beliefs (different agents have different hypotheses about the behavior of opponents) rather than heterogeneity in preferences (different attitudes towards lying) that drive the results.

It would be useful to construct tests that distinguish between lying cost models and level- $k$ models. Doing so would require a careful statement of the competing models. There are many ways to include lying costs (or, more generally, heterogeneous preferences) into communication models. There are also many ways to incorporate heterogeneous levels of strategic sophistication. For example, level- $k$ models need not predict that the existence of a common language chances behavior, but variations of these models are compatible with these predictions.

One approach might be to look at a variety of non-strategic problems (where it may be easy to control for different levels of strategic sophistication) as in Gibson, Tanner, and Wagner (forthcoming).

\section{Do Noisy Channels Improve Outcomes?}

It is now widely known that adding structure to simple cheap-talk game can expand the set of equilibrium outcomes. Myerson (1991) points out that a mediator can improve outcomes. Blume, Board, and Kawamura (2007) and Krishna and Morgan (2004) describe how noisy channels or adding an additional rounds of communication can lead to equilibrium outcomes that are ex ante Pareto superior to all equilibria in a simple cheap-talk game. Randomized response survey techniques (Ljungqvist (1993) and Warner (1965)) use noisy channels to obtain information from informed agents who may be reluctant to make honest reports with the communication channel was perfect. ${ }^{3}$

These studies identify non-partition equilibria. It may be interesting to see if people play these equilibria. A related question is whether removing structure from the communication stage (by allowing subjects to exchange arbitrary messages for a fixed length of time prior to the time when the Receiver selects an action) would change outcomes.

\footnotetext{
${ }^{3}$ Blume, Lai and Lim (2012) provide a strategic model of randomized response procedures and some experimental results.
} 


\section{$5 \quad$ Will Players Add Noise to Their Messages?}

The observation that adding noise may improve outcomes suggests that Senders or Receivers would voluntarily pay to add noise to the communication channel. Blume, Board, and Kawamura (2007) illustrate how it is possible that both Sender and Receiver will prefer a communication system in which the Receiver fails to get the Sender's message with positive probability. It should be straightforward to construct finite games with this property.

In an experimental setting, one can compare the performance of populations who play games that vary only in the degree of noise in the channel. Also, one could modify the game to permit a player to select the quality of the channel prior to sending messages. Would the Receiver prefer to have a noisy channel if it were an option? Would he pay for the option? One can ask these questions of the Sender (both before and after she learns her private information). The results may depend on whether the communication is structured and abstract (so that noise enters messages through a mechanism described by the experimenter and controlled by the Sender) or natural (so that the Sender inserts noise into messages by making imprecise statements in a natural language).

\section{Do Liars Self Select in the Labor Market?}

Both level- $k$ and lying cost theories emphasize that populations are heterogeneous. Outside the lab, one expects that heterogeneous agents would self select, picking vocations that are suited to their tastes. It would be nice to see how members of different professions behave in different settings. Would sales people be more likely to lie than college students? Would this behavior vary with framing of the communication (perhaps with salespeople more likely to lie about product characteristics and students more likely to lie about classroom performance)?

I would like to see a design that induces selection in the laboratory and then tests to see if the selection changes behavior. The selection phase could be as simple as giving the Sender or Receiver the option of participating in a communication game (or receiving a certain payoff). Would adding this possibility reduce communication? How does the effect change when the other player has information about whether the opponent had the option to participate? Would a player wish to publicize a decision to opt in?

\section{Equilibrium Selection with Multiple Senders}

The literature (starting with Krishna and Morgan (2001)) observes that it is often possible to construct fully revealing equilibria with two Senders even when full revelation is not possible when there is one informed agent. This is the case for the game below, with is a modification of the game in Section 1. Make two changes. Assume that when the Sender dines out, she prefers to dine in the same restaurant as the Enemy and add an additional action. (The Receiver is willing to take out food from the Italian restaurant if confident that the Sender's co-worker is not there. The Sender hates take out food.) 


\begin{tabular}{|c||c|c|c|c|}
\hline & Home & Italian & French & Take Out \\
\hline \hline French & 10,0 & 0,5 & $5,-10$ & $-1,4$ \\
\hline Italian & 10,0 & $5,-10$ & 0,5 & $-1,-6$ \\
\hline
\end{tabular}

With one Sender, the only equilibrium outcome is the babbling outcome in which the players stay at home. With two Senders, there is an equilibrium in which the Senders honestly report the Enemy's location (and the Receiver best responds), but the Receiver orders take out when the reports of the Senders disagree. Under what circumstances would experimental subjects play the fully revealing equilibrium? How would the results change if there were more than two Senders?

There is an experimental literature that investigates multi-dimensional cheap-talk with multiple Senders. These papers seek to identify if the fully revealing equilibrium identified by Battaglini (2002) is a good prediction. Lai, Lim, and Wang (2011) conducts experiments on multi-dimensional cheap talk with two Senders. The paper demonstrates that adding a Sender increases the frequency of informative equilibria, but also demonstrates that subjects rarely play fully revealing equilibria that depend on extreme specifications of out-of-equilibrium behavior. Vespa and Wilson (2012) is another experimental study of multi-dimensional cheap talk. This paper demonstrates that adding more senders increases communication in simple setting where each Sender has common interest with the Receiver along a dimension (and different Senders agree with the Receiver along different dimensions). Experimental subjects are less likely to communicate effectively when the distribution of biases is more complicated. These papers strongly suggest that the existence of fully revealing equilibria does not guarantee that full revelation is a good prediction. It would be useful to investigate the extent to which full revelation depends on the degree to which the Sender and Receiver have common preferences over equilibria.

\section{State Independent Communication}

Chakraborty and Harbaugh (2010) study a model of multi-dimensional cheap talk in which the informed Sender's preferences are state independent. They show that informative equilibria exist in this environment. Two features of the equilibria may seem curious. First, because the Sender's preferences are state independent, the Sender will be indifferent between sending the message specified by the equilibrium and some other message in any non-babbling equilibrium. This may create a way to distinguish between explanations that assume heterogeneity of preferences and those that depend on heterogeneous beliefs. Other-regarding preferences or lying costs may break indifference in a systematic way, while different levels of strategic sophistication may yield different implications.

Second, depending on the preferences of the Sender, the Sender may prefer the babbling equilibrium to more informative equilibria. (Communication benefits the Sender if the Sender's preferences are strictly quasiconvex and hurts the Sender if her preferences are strictly quasiconcave.) The first aim of an experiment would be to test whether nontrivial communication arises when the Sender has state-independent preferences. The 
second aim would be to see if the amount of communication varies with the shape of the Sender's preferences. My guess is that communication be observed less frequently when the Sender has quasiconvex preferences.

It should be straightforward to construct games in which informative equilibria exist to investigate whether the Sender will send informative messages even when she does not strictly gain from doing so and, if so, whether the tendency to communicate depends on the existence of ex ante gains from communication. As in other examples, the way in which messages are described is likely to influence the results.

\section{Multiple Audiences}

Does the existence of multiple audiences influence a Sender's willingness or ability to communicate fully? Farrell and Gibbons (1989) introduce a model in which there are two Receivers and a single informed Sender. The Sender has a binary-valued piece of private information. Each Receiver takes an action from a two-point set. Farrell and Gibbons assume that the Sender has separable preferences over the two actions and that the Receivers care only about their own action and the state. They compare equilibria in the game in which the Sender can send private messages to each Receiver to the game in which the Sender makes a public announcement. They identify situations in which a revealing equilibrium exists with public communication but does not exist for one of the games in which the Sender speaks privately to the Receiver.

Farrell and Gibbons theoretical analysis does not consider three things.

If there are behavioral types, increasing the size of the audience may discipline the Sender. Is it possible that adding a non-strategic observer will increase the rate of informative communication? There is a substantial literature on audience effects (for example, Andreoni and Bernheim (2007), Kurzban, DeScioli, and O'Brien (2007), and Rockenbach Milinski (2011)), but I am aware of no study that identifies audience effects in communication games.

Farrell and Gibbons do not discuss the possibility that the Sender can decide whether to communicate in public or in private.

Farrell and Gibbons do not consider the possibility that the Receiver's preferences depend on the action of the other Receiver. Take a situation in which the Receiver can take one of three actions. The first Receiver and the Sender have common preferences. The first Receiver wants to make his action to the state, but if he is uncertain about the state, he takes a third, neutral action. The second Receiver wants to do what the first Receiver does, but has a weak preference for taking the neutral action. Concretely, assume that $A=\{-1,0,1\}$ and $\Theta=\{-1,1\}$ and the two states are equally likely ex ante. The preferences are

$$
\begin{gathered}
U^{S}\left(r_{1}, r_{2}, \theta\right)=-\left(r_{1}-\theta\right)^{2}-\left(r_{2}-\theta\right)^{2}, \\
U^{R_{1}}\left(r_{1}, r_{2}, \theta\right)=-\left(r_{1}-\theta\right)^{2}-\left(r_{2}-\theta\right)^{2},
\end{gathered}
$$

and 


$$
U^{R_{2}}\left(r_{1}, r_{2}, \theta\right)=-\left(r_{1}-r_{2}\right)^{2}-a r_{2}^{2}
$$

where $a>0$ is small. In this setting, there exists a revealing equilibrium with public or with private communication, but it is plausible to conjecture that the second Receiver would be more likely to set $r_{2}=0$ if informed in private.

Checking to see whether adding a non-strategic Receiver increases the frequency of informative communication provides a clean check for the existence of behavioral Senders. If there are behavioral Senders, then there may be self selection when given an opportunity to choose private or public communication. Finally, creating interdependencies provides information about what makes communication credible.

There is (to my knowledge) only one existing experimental study of cheap talk with two audiences. Battaglini and Makarov (2011) conduct experiments on environments identical to those considered in Farrell and Gibbon's paper. Adding an additional Receiver influences the Sender's behavior in directions predicted by theory (for example, increasing communication when the additional Receiver's preferences are aligned with the Sender's), but the experimental games are insufficiently rich to test the hypothesis that adding a neutral Receiver may discipline the Sender.

\section{Multiple Games}

In many common situations involving communication the Sender and Receiver have aligned interests. It is probably safe to say that conflict is even rarer for children when they acquire language. Perhaps the tendency to observe laboratory subjects who make more informative statements and are more credulous than game theory predicts stems from the fact that people learn to communicate in common-interest settings, they participate primarily in common-interest settings in natural settings, and it may not be worth the effort to deviate from simple, general, and reasonable rules of behavior ("tell the truth," "take statements at face value").

One cannot hope to simulate developmental processes in a laboratory setting, but perhaps some simple treatments could shed light on how people behave in an environment in which games with conflict are mixed with common interest games. I describe two ideas.

First, one can imagine having agents play two different games (with different partners). One game should be a coordination game in which a fully revealing equilibrium exists. In the other game, there is conflict. (Ideally it would be a game in which there are multiple, partially pooling equilibria.) One can prime some subjects by having them first play the coordination game and then the game with conflict, while another group plays in a different order. Will experience change the behavior of the players? Will information about the experience of opponents (consisting of the types of games played, rather than their behavior) influence behavior?

Second, one can tell Senders that they will face one of two games, one with conflict and one without. Suppose that the prior distribution is such that, without further information, a fully revealing equilibrium would exist. Permit the Sender to learn the type of the game (at a cost). Would the Sender pay the cost? Would the existence of the cost change behavior? 
To my knowledge, there have been no experiments of this kind. There is a related literature on learning that asks whether agents transfer knowledge from one environment to another.Ho, Camerer, and Weigelt (1998) argue that players with experience in one $p$-beauty contest converge more quickly than inexperienced players in similar games with different values of $p$. Cooper and Kagel (2008) demonstrate that cross-game learning arises in signaling games. Haruvy and Stahl (2009) show that learning rules of behavior (in particular, learning not to play a strictly dominated strategy) can operate over different games. Subjects with experience in one normal-form game learn more quickly in other games. These papers provide ample evidence that players connect experience in one situation to other situations. I am particularly interested in whether the outcome of the learning process depends on the mix of games and whether there are situations in which agents overgeneralize, applying rules of behavior that work in one setting to settings in which they are no longer appropriate or selectively restrict their opportunities to learn. 


\section{References}

Andreoni, J., And B. D. Bernheim (2007): "Social Image and the 50-50 Norm: A Theoretical and Experimental Analysis of Audience Effects," Econometrica, 77(5), $1607-1636$.

Battaglini, M. (2002): "Multiple Referrals and Multidimensional Cheap Talk," Econometrica, 70(4), 1379-1401.

Battaglini, M., and U. Makarov (2011): "Cheap Talk with Multiple Audiences: an Experimental Analysis," Discussion paper, Princeton University.

Blume, A., O. J. Board, And K. Kawamura (2007): "Noisy talk," Theoretical Economics, 2(4), 395-440.

Blume, A., D. V. DeJong, Y. Kim, and G. B. Sprinkle (1998): "Experimental evidence on the evolution of meaning of messages in sender-receiver games," The American Economic Review, 88(5), 1323-1340.

Blume, A., D. V. DeJong, Y. Kim, and G. B. Sprinkle (2001): "Evolution of communication with partial common interest," Games and Economic Behavior, 37(1), $79-120$.

Blume, A., E. K. Lai, and W. Lim (2012): "A Game Theoretic Approach to Randomized Response: Theory and Experiment," Discussion paper, Leheigh University.

CAI, H., AND J. T. WANG (2006): "Overcommunication in strategic information transmission games," Games and Economic Behavior, 56(1), 7-36.

Chakraborty, A., and R. Harbaugh (2007): "Comparative Cheap Talk," Journal of Economic Theory, 132(1), 70-94.

82.

Charness, G., and M. Dufwenberg (2006): "Promises and partnership," Econometrica, 74(6), 1579-1601.

Chen, Y. (2006): "A Non-Fully Rational Model of Communication," Discussion paper, Arizona State University.

Chen, Y., N. Kartik, and J. Sobel (2008): "On the Robustness of Informative Cheap Talk," Econometrica, 76(1), 117-136.

Chung, W., and R. Harbaugh (2012): "Biased Recommendations," Discussion paper, Indiana University.

Cooper, D. J., And J. H. KAGEL (2008): "Learning and transfer in signaling games," Economic Theory, 34(3), 415-439. 
Cooper, R. W., D. V. DeJong, R. Forsythe, and T. W. Ross (1992): "Communication in Coordination Games," The Quarterly Journal of Economics, 107(2), 739-771.

Crawford, V. P., And J. Sobel (1982): "Strategic Information Transmission," Econometrica, 50(6), 1431-1451.

Farrell, J., And R. Gibbons (1989): "Cheap talk with two audiences," The American Economic Review, 79(5), 1214-1223.

Gibson, R., C. Tanner, and A. Wagner (forthcoming): "Preference for Truthfulness: Heterogeneity Among and Within Individuals," American Economic Review.

Gneezy, U. (2005): "Deception: The Role of Consequences," American Economic Review, 95(1), 384-394.

Gordon, S. (2011): "Iteratively Stable Cheap Talk Equilibrium," Discussion paper, Université de Montréal.

Haruvy, E., and D. O. Stahl (2009): "Learning Transference Between Dissimilar Symmetric Normal-Form Games," Discussion paper, University of Texas.

Ho, T.-H., C. Camerer, and K. Weigelt (1998): "Iterated dominance and iterated best response in experimental 'p-beauty contests'," The American Economic Review, 88(4), 947-969.

Hurkens, S., and N. Kartik (2009): "Would I Lie to You? On Social Preferences and Lying Aversion," Experimental Economics, 12(2), 180-192.

Kartik, N. (2009): "Information Transmission with Almost-Cheap Talk," Review of Economic Studies, 76(4), 1359-1395.

Kartik, N., And J. Sobel (2012): "Effective Communication in Cheap-Talk Games," Discussion paper, UCSD.

Krishna, V., and J. Morgan (2001): "Asymmetric Information and Legislative Rules: Some Amendments," American Political Science Review, 95(2), 435-452.

_ (2004): "The art of conversation: eliciting information from experts through multi-stage communication," Journal of Economic Theory, 117(2), 147-179.

Kurzban, R., P. DeScioli, and E. O'Brien (2007): "Audience effects on moralistic punishment," Evolution and Human Behavior, 28, 75-84.

Lai, E. K., W. Lim, and J. T. Wang (2011): "Experimental Implementations and Robustness of Fully Revealing Equilibria in Multidimensional Cheap Talk," Discussion paper, Lehigh University.

LJUNGQVist, L. (1993): "A unified approach to measures of privacy in randomized response models: A utilitarian perspective," Journal of the American Statistical Association, 88(421), 97-103. 
Myerson, R. B. (1991): Game Theory: Analysis of Conflict. Harvard University Press, Cambridge, Massachusetts.

Rockenbach, B., And M. Milinski (2011): "To qualify as a social partner, humans hide severe punishment, although their observed cooperativeness is decisive," Proceedings of the National Academy of Sciences, 108(45), 18307-18312.

Vespa, E., And A. J. Wilson (2012): "Communication with Multiple Senders and Multiple Dimensions: An Experiment," Discussion paper, New York University.

Wang, J. T., M. Spezio, and C. F. Camerer (2010): "Pinocchio's Pupil: Using Eyetracking and Pupil Dilation To Understand Truth-telling and Deception in Games," American Economic Review, 100(3), 984-1007.

Warner, S. L. (1965): "Randomized Response: A Survey Technique for Eliminating Evasive Answer Bias," Journal of the American Statistical Association, 60(309), 63-69. 